\title{
"SHOUT" to improve the quality of care delivered to patients with acute kidney injury at Great Western Hospital
}

Paul Brady, James Gorham, Angeliki Kosti, William Seligman, Alona Courtney, Karolina Mazan, Stuart Paterson, Steve Ramcharitar, Badri Chandrasekaran, Mark Juniper, Mala Greamspet, Jessica Daniel, Sue Chalstrey, ljaz Ahmed, Tanaji Dasgupta

Great Western Hospital

\begin{abstract}
Acute kidney injury (AKI) affects up to $20 \%$ of all patients admitted to hospital, and is associated with a higher risk of adverse clinical outcomes, increased healthcare costs, as well as long term risks of chronic kidney disease and end stage renal failure. The aim of this project was to improve the quality of care for patients with AKI admitted to the acute medical unit (AMU) at the Great Western Hospital (GWH). We assessed awareness and self reported confidence among physicians in our Trust, in addition to basic aspects of care relevant to AKI on our AMU. A multifaceted quality improvement strategy was developed, which included measures to improve awareness such as a Trust wide AKI awareness day, and reconfiguring the admission proforma on our AMU in order to enhance risk assessment, staging, and early response to AKI. Ancillary measures such as the dissemination of flashcards for lanyards containing core information were also used. Follow up assessments showed that foundation year one (FY1) doctors' self reported confidence in managing AKI increased from 2.8 to 4.2 , as measured on a five point Likert scale $(P=0.0003)$. AKI risk assessment increased from $13 \%$ to $57 \%(P=0.07)$ following a change in the admission proforma. Documentation of the diagnosis of $A K I$ increased from $66 \%$ to $95 \%(P=0.038)$ among flagged patients. Documentation of urine dip results increased from $33 \%$ to $73 \%(P=0.01)$, in addition to a rise in appropriate referral for specialist input, although this was not statistically significant. Our results suggest that using the twin approaches of improving awareness, and small changes to systemic factors such as modification of the admission proforma, can lead to significant enhancements in the quality of care of patients with AKI.
\end{abstract}

\section{Problem}

Acute kidney injury (AKI) is an increasingly common concurrent complication among inpatients, particularly in the elderly, and those with multiple comorbidities. Studies show it is often avoidable, poorly recognised, and suboptimally managed.[1] Consequently, AKI is associated with higher healthcare costs and adverse clinical outcomes. There is an urgent need to improve the quality of care in patients with AKI by improving risk assessment, early identification, and response.

\section{Background}

AKI refers to an acute deterioration in renal function, which can range from mild elevations in serum creatinine to severe anuric renal failure with uraemia and its complications. AKI is very common, and is present in approximately $13 \%$ to $18 \%$ of acute admission. It is well recognised that AKI is associated with higher risks of inpatient mortality, increased length of hospital stay, long term mortality, as well as the risks of chronic kidney disease and end stage renal failure. It is associated with substantial healthcare costs, accounting for almost $1 \%$ of all NHS spending.[2, 3]

Most patients with $\mathrm{AKI}$ are managed by healthcare professionals in a wide range of specialties. Previous studies have shown there is considerable room for improvement in prevention, recognition, and early and subsequent management of patients with AKI.[1]

The National Institute for Health and Care Excellence (NICE) clinical guideline 169 was published in 2013, and highlights the role of prevention, early recognition, and identification of an underlying cause in the management of AKI. This guideline also emphasises the need to support patients and their carers with information, and sets out this advice for the non-specialist who is likely to manage AKI in a number of different settings.[4]

Electronic flagging of laboratory results that might suggest a diagnosis of $\mathrm{AKI}$ is used across the NHS, using a pre-specified algorithm as outlined in a recent National Patient Safety Agency (NPSA) alert, in concert with AKI management pathways.[5] At the Great Western Hospital (GWH), electronic flagging of AKI and a management pathway including an AKI care bundle have been operational since August 2013. The care bundle is derived from the core recommendations in NICE guidance, and uses the mnemonic "SHOUT," denoting sepsis, hypovolaemia, obstruction, urine analysis, and a review of any nephrotoxic factors (see Appendix 1).

The aim of this project was to improve the quality of care among inpatients in our hospital with AKI admitted to our acute medical unit (AMU), by:

1. Improving awareness among clinicians

2. Improving recognition of AKI

3. Encouraging the use of tools for risk assessment, with a view to prevention

4. Increasing the use of a clinical tool designed to systematise early management and appropriate referral for specialist input by using the SHOUT AKI care bundle. 
See supplementary file: ds6143.pdf - "Appendix 1-GWH SHOUT AKI care bundle"

\section{Baseline measurement}

\author{
Assessing awareness
}

A survey was carried out at the weekly grand round meeting to generate a focused quality improvement strategy on enhancing AKI awareness and management, allowing us to assess self perceived confidence and awareness of the Trust care bundle in identification and management of AKI. This initial survey included 13 consultants, 10 registrars, 15 senior house officers (SHOs), and 13 foundation year one (FY1) doctors.

A Likert scale was used to enable doctors to grade their self perceived confidence in managing AKI from zero (no confidence) to five (very confident). Confidence in managing AKI varied considerably at different levels of seniority, with consultants and registrars having highest confidence in managing AKI (mean 4.2 for both consultants and registrars), followed by SHOs and FY1 doctors averaging 3.7 and 2.8 respectively (see graph 1.1 ).

Knowledge of the clinical indications for specialist referral in AKI as specified in NICE guidelines was tested among these clinicians. Consultants and registrars scored the highest, with $62 \%$ and $60 \%$ of participants answering this question correctly respectively. $\mathrm{SHOs}$ had a $53 \%$ correct response rate. Significantly, FY1s scored the lowest, with only $8 \%$ of these doctors knowing when to refer to a nephrologist (graph 1.2).

A similar trend was observed in questions pertaining to awareness of the SHOUT AKI care bundle, with $100 \%$ of consultants being aware compared to only $23 \%$ of $F Y 1$ doctors (graph 1.3 ).

Assessment of clinical care

A baseline audit was undertaken at GWH in November 2014, with the goal of assessing the extent of risk assessment and basic management steps relevant in a patient identified as having AKI. AKI was defined according to Kidney Disease Improving Global Outcomes (KDIGO) criteria.[6] A sample of 30 consecutive patients presenting to the acute medical admissions ward with AKI was selected from the electronic laboratory system. The NICE CG169 AKI clinical audit toolkit was used to audit clinical care. This included recording a risk assessment of, and where flagged, the diagnosis and cause of AKI. In addition, other key aspects such as performing a urine dipstick, recording fluid balance, adherence to NICE guidance regarding imaging of the urinary tract, and referral were assessed.

At baseline, of all flagged patients, $13 \%$ were documented as having had an assessment for AKI risk factors on admission (graph 2.1). Sixty six percent of patients had AKI documented as a diagnosis within 24 hours (graph 2.2). Of those patients who had an indication for referral to nephrology, $42 \%$ were referred for specialist input (graph 2.3). The SHOUT AKI care bundle was completed in $3 \%$ of cases (graph 2.4 ), and urinalysis results were documented in
$33 \%$ of cases (graph 2.5).

See supplementary file: ds6138.png - "Graph 1 - Doctor survey results"

\section{Design}

An AKI awareness group consisting of seven junior doctors carried out this quality improvement project between November 2014 and May 2015. Interventions were derived from the primary drivers, which encompassed promotion of NICE guidelines, enhancement in staff knowledge and awareness of AKI, and promotion of screening and early recognition of $\mathrm{AKI}$ in clinical practice (figure 1).

In order to assess awareness, an initial survey was carried out in November 2014 as earlier described. This was repeated in March 2015 ( $n=49$; 12 consultants, 13 registrars, 13 SHOs, and 11 FY1 doctors), in order to reassess the impact of our interventions.

Moreover, in order to assess the impact of our interventions on clinical care, The NICE CG169 AKI clinical audit toolkit was implemented in the baseline audit in November 2014, with a sample of 30 consecutive patients presenting with AKI to the AMU. This process was repeated in succeeding PDSA cycles, with an identical sample size of 30 on each occasion.

The first PDSA cycle was completed in March 2015 following a multifaceted quality improvement strategy. This included a Trust AKI awareness day coinciding with World Kidney Day, supported by a Trust wide information campaign. The latter included flyers on all hospital wards, mass mailings to all Trust clinical staff, and reconfiguration of the Trust intranet front page, bringing together all relevant information and an $\mathrm{AKI}$ video presentation featuring clinical leaders in the Trust. This was complemented by the introduction of an AKI screening and response section embedded within the clerking proforma, for admissions to the acute medical admissions unit.

In May 2015 a second PDSA cycle was completed, which encompassed an AKI awareness lecture at the weekly grand round, and reinforcement of the core principles of the SHOUT AKI care bundle; and referral criteria on flashcards that could be attached to lanyards.

In terms of statistical analysis, nonparametric categorical data from November 2014 was compared to data from April 2015 using the Mann-Whitney $U$ test. A two sided $P$ value of $<0.05$ was used as a threshold to denote statistical significance. Results were analysed using Stata 14.

\section{Strategy}

Awareness and education strategy: AKI awareness day [PDSA Cycle 1]

An AKI awareness day coinciding with World Kidney Day was organised, with the aims of raising general awareness, providing 
specific education to various groups of the multidisciplinary team, and to promote the use of the SHOUT AKI care bundle. Methods used to achieve this objective included an audiovisual flagship presentation featuring the clinical leaders in the Trust, which featured on the Trust intranet along with coverage in the local press. In addition, AKI themed posters were presented, and simulation based methods employed to deliver the core messages.

Modification of admission proforma to drive improvement in clinical care [PDSA Cycle 1]

An AKI screening and response tool was introduced into the AMU proforma in February 2015. The components of this tool consist of a series of individual sections that help facilitate the identification and management of AKI (figure 2). Three sections were designed in accordance with NICE guidance, and included the following:

1. AKI risk assessment: this section contained $14 \mathrm{AKI}$ risk factors as described in NICE CG192, to assist the clinician in recognising the potential cumulative effect of multiple risk factors

2. AKI identification and staging: a designated section in which the patient's current and baseline creatinine can be recorded, in order to confirm and stage AKI

3. AKI clinical care: sections requiring recording of urinalysis results, and indication of whether a SHOUT AKI care bundle was being completed, followed section two on the admissions program.

Educational session [PDSA Cycle 2]

In April 2015 a second PDSA cycle commenced, and this was initiated by delivering an AKI awareness lecture at the weekly grand round. The aim of this event was to reiterate the message delivered at the AKI awareness day. The lecture encompassed an AKI educational presentation delivered by a consultant nephrologist, and was followed by a brief demonstration of how to use the AKI screening tool.

Dissemination of written guidelines [PDSA Cycle 2]

In order to increase awareness and accessibility of the SHOUT AKI care bundle, the care bundle was first modified and printed onto laminated pocket sized flash cards, which could be attached to a lanyard and distributed to all junior doctors working at GWH.

See supplementary file: ds6144.png - "Figure 1: Driver Diagram, Figure 2: Modified AMU proforma"

\section{Post-measurement}

\section{Awareness}

A statistically significant increase was found in FY1 doctors' self reported confidence in managing $\mathrm{AKI}$, from 2.8 to 4.2 as measured on a five point Likert scale ( $P=0.0003)$ (graph 1.1). In terms of knowledge on indications for specialist referral as per current NICE guidelines, all groups of doctors performed better on retesting (graph 1.2). In particular, $73 \%$ of $F Y 1$ doctors answered this question correctly on retesting, compared to eight percent in the original survey $(\mathrm{P}=0.69)$. There was also an increase in awareness of the SHOUT AKI care bundle among registrars, SHOs, and FY1 doctors (graph 1.3). FY1 doctors in particular demonstrated a statistically significant improvement, from $23 \%$ at baseline to $91 \%$ following the first PDSA cycle $(P=0.026)$.

\section{Clinical care}

The baseline audit performed in November 2014 demonstrated that just $13 \%$ of patients had a documented risk assessment for $\mathrm{AKI}$ at admission. This increased to $57 \%(P=0.07)$ following the introduction of the AKI risk assessment tool into the AMU admissions proforma (graph 2.1). Documentation of the diagnosis of AKI increased from $66 \%$ in the baseline audit to $95 \%$ among all flagged patients following PDSA cycle 2 in April $(P=0.038)$ (graph 2.2). In terms of appropriate referral to nephrology based on NICE guidelines, this increased from $42 \%$ in the baseline audit in November 2014 to $71 \%$ in April 2015 following PDSA cycle two ( $P=0.25)$ (graph 2.3). The implementation of the AKI care bundle increased from three percent in November 2014 to $56 \%$ in April 2015 following PDSA cycle two ( $P=0.35$ ) (graph 2.4). In conjunction with this was a statistically significant increase in the documentation of urine dip results, from $33 \%$ to $73 \%(P=0.01)$ following PDSA cycle two compared to the baseline audit (graph 2.5).

See supplementary file: ds6140.png - "Graph 2: Clinical Care QI project Results"

\section{Lessons and limitations}

Due to the number of patients studied in each cycle, it was not possible to detect statistically significant differences in some of the outcomes measured. Furthermore, this study was not powered to detect statistically significant changes in patient morbidity or mortality following the implementation of these quality improvement initiatives.

Results in this project were analysed by carrying out statistical tests comparing data generated in the baseline audit with data generated in the final PDSA cycle. A more robust strategy would have been to use information gathered throughout the project to produce run charts, thereby enabling data to be analysed via statistical process control (SPC).

Many of the aims of this project did not follow SMART rules, ie specific, measurable, achievable, realistic, and time bound. Two examples of this include improving awareness and improving recognition. This was a significant limitation, as it meant that in many respects the project did not fully embrace the philosophy of quality improvement in contrast to audit. A more robust strategy would therefore have been to make small regular measurements and assessment of how interventions made a difference, versus natural variation in performance.

\section{Conclusion}




\section{BMJ Quality Improvement Reports}

Delivering a set of interventions aimed at improving awareness, and access to clinical tools to systematise care, resulted in statistically significant improvements in a recorded diagnosis of $\mathrm{AKI}$, and a statistically significant higher percentage of patients having urine examined in the context of an admission with AKI. The National Confidential Enquiry into Patient Outcome and Death (NCEPOD) report reminds us of the need for better recognition and response to AKI. The essential drivers of change are likely to be improved education, systemic checks for early identification of $\mathrm{AKI}$, and in turn a number of systematic responses in order to facilitate treatment and prevention of AKI.

Owing to the success of the AKI awareness day, organised as a flagship event for this quality improvement project, the renal team at GWH are now committed to making this an annual staged event to coincide with World Kidney Day. In conjunction with the modified medical clerking proforma this will help to ensure that AKI awareness is sustained at GWH. Future initiatives may include linking the process of flagging to the use of an electronic version of the AKI care bundle, and using historical discharge codes and comorbidity scores to inform risk assessment at admission.

\section{References}

1. National Confidential Enquiry into Patient Outcome and Death. Adding Insult to Injury: A review of the care of patients who died in hospital with a primary diagnosis of acute kidney injury (acute renal failure). 2009. London: National Confidential Enquiry into Patient Outcome and Death. www.ncepod.org.uk/2009report1/Downloads/AKI report.pdf

2. Selby NM, Crowley L, Fluck RJ, et al. Use of electronic results reporting to diagnose and monitor AKI in hospitalized patients. Clin J Am Soc Nephrol 2012;7(4):533-40.

3. Kerr M, Bedford M, Matthews B, O'Donoghue D. The economic impact of acute kidney injury in England. Nephrol Dial Transplant 2014;29(7):1362-8.

4. National Institute for Health and Care Excellence. Acute kidney injury: prevention, detection and management. (Clinical guideline 169.) 2013. http://guidance.nice.org.uk/CG169.

5. NPSA. Patient Safety Alert Stage Three: Directive. Standardising the early identification of Acute Kidney Injury. 2014. https://www.england.nhs.uk/wpcontent/uploads/2014/06/psa-aki.pdf

6. Kidney Disease: Improving Global Outcomes (KDIGO) Acute Kidney Injury Work Group. KDIGO clinical practice guideline for acute kidney injury. Kidney Int Suppl 2012;2(1):1-138.

\section{Declaration of interests}

Nothing to declare

\section{Acknowledgements}

Dr Amanda Pegden, Dr Robert Hughes, Dr Gea Gonzalez

\section{Ethical approval}

Ethical approval was not sought for this project, as it was deemed an improvement study, and not a study on human subjects, and local policy meant that ethical approval was not required. 